\title{
A GENERAL TOOL FOR DETERMINING THE ASYMPTOTIC SPECTRAL DISTRIBUTION OF HERMITIAN MATRIX-SEQUENCES
}

\author{
Carlo Garoni, Stefano Serra-Capizzano and Paris Vassalos
}

Abstract. We consider sequences of Hermitian matrices with increasing dimension, and we provide a general tool for determining the asymptotic spectral distribution of a 'difficult' sequence $\left\{A_{n}\right\}_{n}$ from the one of 'simpler' sequences $\left\{B_{n, m}\right\}_{n}$ that approximate $\left\{A_{n}\right\}_{n}$ when $m \rightarrow \infty$. The tool is based on the notion of an approximating class of sequences (a.c.s.), which was inspired by the work of Paolo Tilli and the second author, and it is applied here in a more general setting. An a.c.s.-based proof of the famous Szegö theorem on the spectral distribution of Toeplitz matrices is finally presented.

Mathematics subject classification (2010): 47B06, 15A60, 15B05. matrices.

Keywords and phrases: Approximating class of sequences, spectral distribution, Szegö theorem, Toeplitz

\section{REFERENCES}

[1] O. Axelsson, G. LindsKog, On the rate of convergence of the preconditioned conjugate gradient method, Numer. Math. 48 (1986) 499-523.

[2] R. Bhatia, Matrix analysis, Springer-Verlag, New York (1997).

[3] A. Böttcher, S. Grudsky, Spectral properties of banded Toeplitz matrices, SIAM, Philadelphia (2005).

[4] A. Böttcher, B. Silbermann, Introduction to large truncated Toeplitz, matrices, Springer-Verlag, New York (1999).

[5] P. J. DAvis, Circulant matrices, $2^{\text {nd }}$ Edition, AMS Chelsea Publishing (1994).

[6] C. Garoni, S. Serra-Capizzano, D. SesAna, Tools for determining the asymptotic spectral distribution of non-Hermitian perturbations of Hermitian matrix-sequences and applications, Integr. Equat. Oper. Theory 81 (2015), 213-225.

[7] G. H. Golub, C. F. VAN LoAn, Matrix computations, $3^{\text {rd }}$ Edition, The Johns Hopkins University Press (1996).

[8] U. Grenander, G. SzEGö, Toeplitz forms and their applications, $2^{\text {nd }}$ Edition, Chelsea, New York (1984).

[9] W. RUdin, Real and complex analysis, $3^{\text {rd }}$ Edition, McGraw-Hill (1987).

[10] S. SERRA-CAPIZZANo, Distribution results on the algebra generated by Toeplitz sequences: a finite dimensional approach, Linear Algebra Appl. 328 (2001) 121-130.

[11] S. SERRA-CAPIZZANO, Spectral behavior of matrix sequences and discretized boundary value problems, Linear Algebra Appl. 337 (2001) 37-78.

[12] S. SERRA-CAPIZZANO, More inequalities and asymptotics for matrix valued linear positive operators: the noncommutative case, Oper. Theory Adv. Appl. 135 (2002) 293-315.

[13] P. TILli, Locally Toeplitz sequences: spectral properties and applications, Linear Algebra Appl. 278 (1998) 91-120.

[14] P. TILli, A note on the spectral distribution of Toeplitz matrices, Linear and Multilinear Algebra 45 (1998) 147-159.

[15] E. E. TyRTYShNikov, A unifying approach to some old and new theorems on distribution and clustering, Linear Algebra Appl. 232 (1996) 1-43. 
[16] N. L. Zamarashin, E. E. TyRTYShnikov, Distribution of eigenvalues and singular values of Toeplitz matrices under weakened conditions on the generating function, Sb. Math. 188 (1997) 11911201. 